\title{
Use of Tomography for Diagnostic Purposes in Primary Ciliary Dyskineasia
}

\author{
J. Brink* and J.L. Carson** \\ *JEOL USA Inc., Peabody MA 01960 \\ **University of North Carolina at Chapel Hill, Chapel Hill NC 27599
}

Tomography has been widely used and recognized as an important tool for structural research in Life Sciences [1,2]. Here, we applied tomography to aid in the diagnosis of primary ciliary dyskineasia (PCD). PCD belongs to a family of genetically hetergeneous disorders of motile cilia with a wide range of phenotypes but to a large degree involve mutations in the gene coding for the cilairy dynein arms [3]. The typical workflow in diagnosing a case suspected of PCD involves scanning large numbers and/or large areas of plastic resin-embedded and conventionally stained thin sections looking for axial cross sections through cilia from which a diagnosis is made. The relatively low occurrence of near-perfect axial cross-sections of cilia has prompted us to investigate whether tomography can be applied to this field and help speeding up the diagnosis. Although tomography has been applied to PCD before [4], it has never found an application as a routine diagnostic tool.

Specimens from cultured epithelium cells from a healthy human subject and a patient with PCD were fixed in $2 \%$ paraformaldehyde with $2 \%$ glutaraldehyde and $0.5 \%$ tannic in phosphate buffer, known to optimally stabilize and fix microtubular structures. Silver sections were post-stained with uranyl acetate and lead citrate for microscopy. Tomography was performed in a JEOL JEM-1400 transmission electron microscope operating at $120 \mathrm{kV}$ using SerialEM to acquire the tilt series ( $+/-60^{\circ}$ at $1.5-2^{\circ}$ intervals) [5]. The tilt series were processed using IMOD [6].

Figure 1 shows a set of image fields of epithelial brush borders from healthy and PCD-cases featuring many cilia. The fields are from the untilted views from the tilt series and normally would not suffice for a diagnosis, as none of the cilia reveal the proto-typical pattern. Figure 2 shows close-ups from the three-dimensional reconstruction generated from tilt data of a healthy individual. This view clearly reveals glancing cross-sections through several cilia. However, the $3 \mathrm{D}$ reconstruction can easily be tilted in the computer to reveal the proto-typical pattern with, among others features, connectivity of the dynein arms clearly revealed. Similarly, figure 3 shows the PCD-case, whereupon tilting of the reconstruction the disconnected dynein arms are now resolved. Important to note here is that the data was obtained in less than 30 minutes per case and the reconstructions were also obtained in under 30 minutes suggesting a diagnosis in under 1 hour. This data shows a proof of concept of applying tomography to diagnosing PCD cases. Support from the Primary Ciliary Dyskineasis Foundation is greatly appreciated.

\section{References}

[1] Frank, J., Electron Tomography: Methods for Three-Dimensional Visualization of Structures in the Cell, $2^{\text {nd }}$ ed., Springer, Albany, NY (2006).

[2] McIntosh, JR., Cellular Electron Tomography in: Methods in Cell Biology Series, Vol. 79, Elsevier, Amsterdam (2007).

[3] Leigh, MW et al., Genetics in Medicine 11 (2009) 473.

[4] O’Toole, E.T. et al., Microsc. Microanal. 14 (Suppl 2) (2008) 1476CD.

[5] Mastronarde, D.N., Microsc. Microanal. 9 (Suppl. 2) (2003) 1182CD.

[6] Kremer J.R. et al., J. Struct. Biol. 116 (1996) 71. 


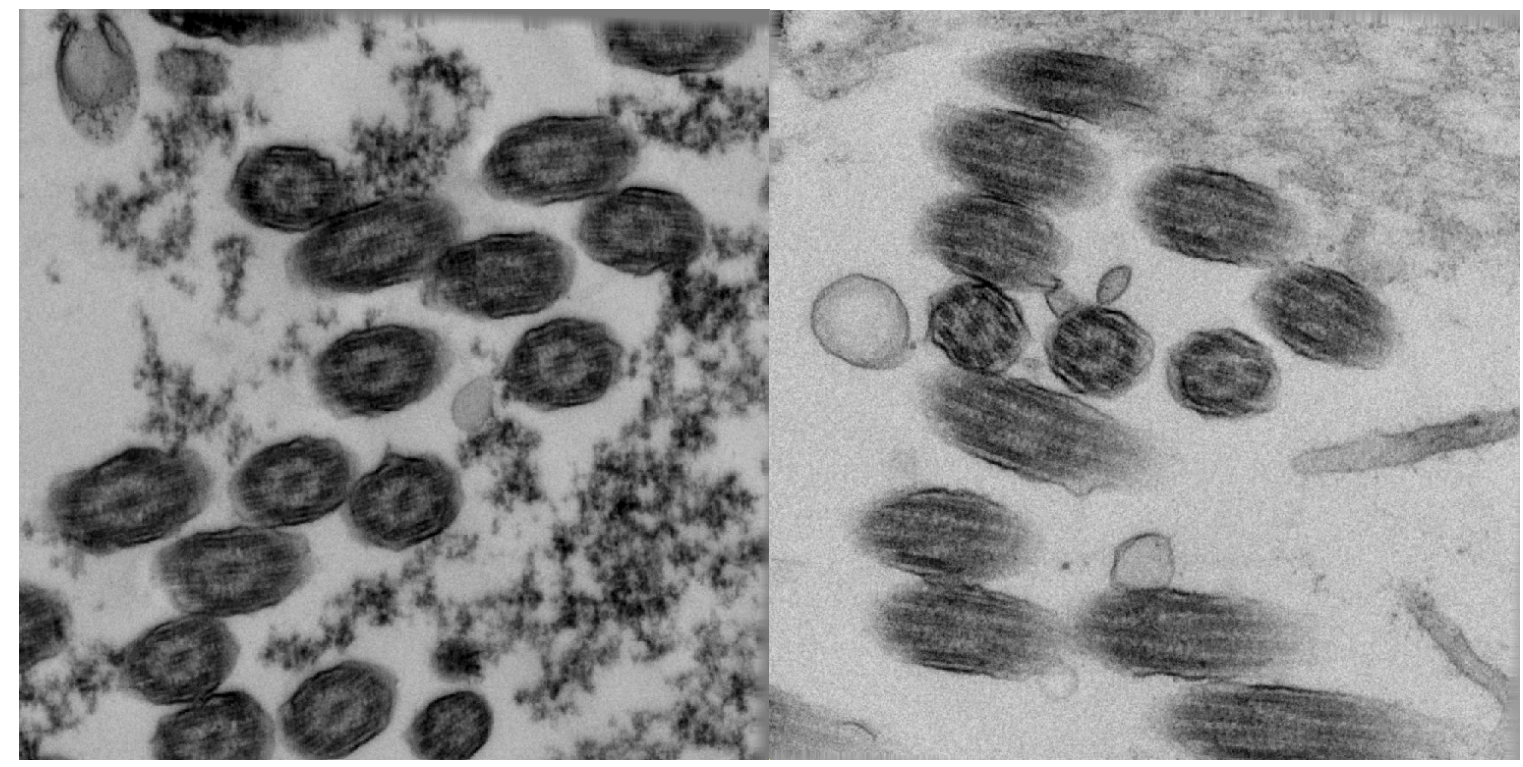

Fig. 1: Untilted views of epithelial brush borders of healthy (left) and PCD-afflicted individuals (right).

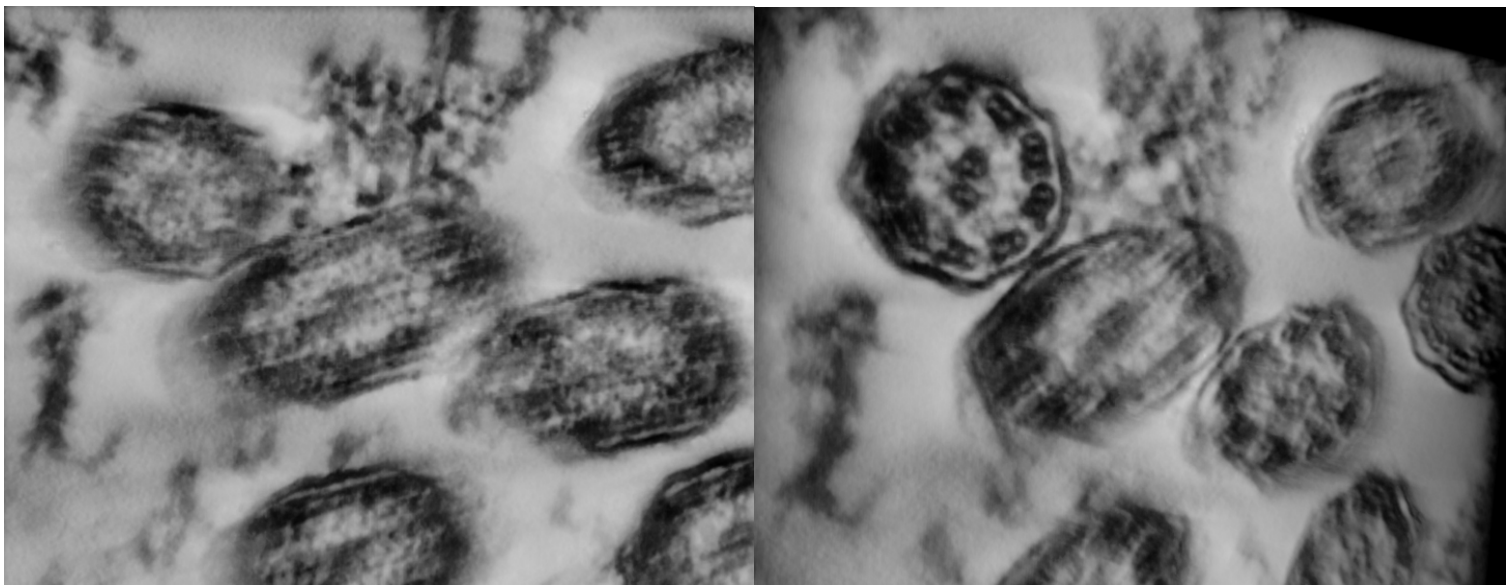

Fig. 2: Close-ups of the reconstruction of cilia of a healthy individual untilted (left) and tilted to reveal the proto-typical view used for diagnostics (right). The tilt of the reconstruction was chosen to reveal the pattern in the upper left cilia.

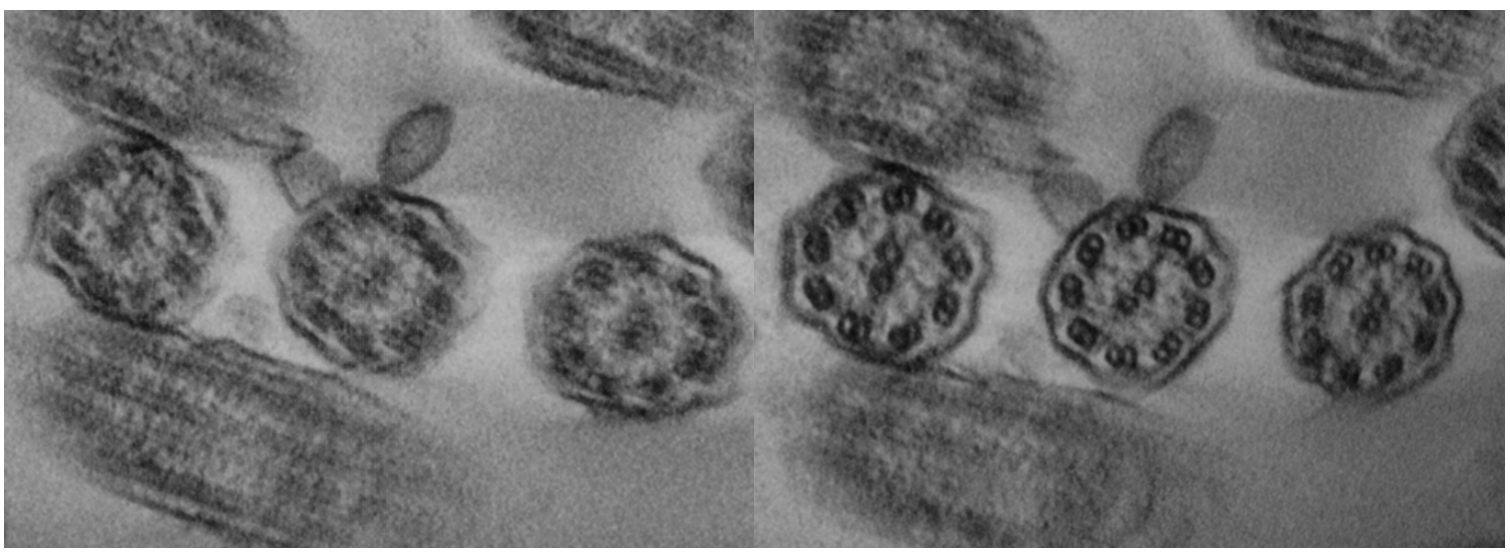

Fig. 3: As in figure 2, but now from a PCD-case. 